See Article page 1239.

\section{Commentary: Treating tricuspid regurgitation: Too much too early or too little too late. Can we find the right balance?}

\author{
Shakirat O. Oyetunji, MD, and Gabriel S. Aldea, MD
}

Surgical tricuspid valve repair had been shown to be safe (no added mortality) and effective when performed at the time of concomitant mitral valve interventions. ${ }^{1,2}$ However, the indications and precise timing of interventions for isolated tricuspid regurgitation (TR) remain to be defined. The tricuspid valve is now the subject of intense renewed interest, prompted by increased recognition that TR is common, frequently clinically silent for years, but its presence is far from benign and associated with a worse prognosis. ${ }^{2-4}$ Better awareness, more precise imaging and quantitation, and a growing spectrum of open surgical as well as catheter-based technologies also refocused this growing interest.

In this issue of the Journal, Fröjd and colleagues ${ }^{5}$ published their experience of the management of TR by comparing 2 time periods, 1989 to 2005 and 2006 to 2015. The study is limited by its small size, inherent selection bias of retrospective studies, and unmeasured confounders such as right ventricular ejection fraction and the presence/degree of pulmonary hypertension. Like others, the authors demonstrated that the incidence of interventions increased, mortality decreased (and was better for lower TR grades), and 5-year survival improved in the most recent era with more repairs being performed. This is attributed to earlier surgery, better patient selection, and improved medical management of right heart failure. As expected, the vast majority of TR interventions were done concomitantly with other cardiac surgery procedures $(89 \%$, with less than 3 isolated TR interventions per year). The authors suggest that

\footnotetext{
From the Division of Cardiothoracic Surgery, University of Washington, Seattle, Wash.

Disclosures: The authors reported no conflicts of interest.

The Journal policy requires editors and reviewers to disclose conflicts of interest and to decline handling or reviewing manuscripts for which they may have a conflict of interest. The editors and reviewers of this article have no conflicts of interest.

Received for publication Dec 12, 2020; revisions received Dec 12, 2020; accepted for publication Dec 14, 2020; available ahead of print Dec 25, 2020.

Address for reprints: Gabriel S. Aldea, MD, University of Washington, Box 356310, 1959 NE Pacific St, Seattle, WA 98915 (E-mail: aldea@uw.edu).

J Thorac Cardiovasc Surg 2021;161:1249-50

$0022-5223 / \$ 36.00$

Copyright (c) 2020 by The American Association for Thoracic Surgery

https://doi.org/10.1016/j.jtcvs.2020.12.062
}

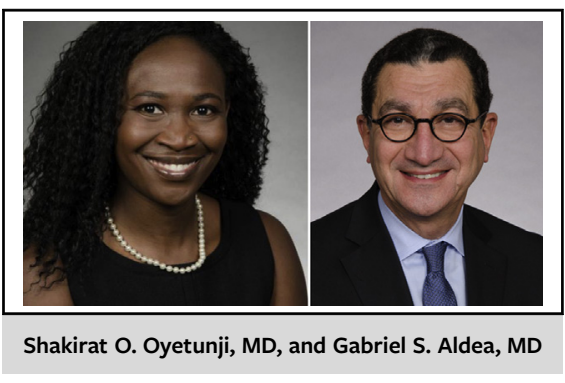

CENTRAL MESSAGE

The tricuspid valve regurgitation

(TR) is the subject of renewed

interest but timing, extent, and

effectiveness of treatment stra-

tegies (particularly for isolated

TR) remain to be defined.

these improved outcomes should prompt consideration for earlier therapy of TR (before onset of right ventricular failure, chronic atrial fibrillation, hepatic or renal dysfunction). These updated procedural and survival outcomes should be the benchmarks for comparison for emerging catheterbased interventions.

While we applaud and support these conceptual suggestions, we have to acknowledge that although earlier interventions would be associated with lower morbidity and mortality (like those for aortic and mitral valve insufficiency pathologies), there is a paucity of prospective randomized data to support interventions at clinically earlier (silent) stages of tricuspid valve pathology, particularly for secondary TR. On the other end of the spectrum, it is unclear whether late interventions for secondary TR in the presence of significant $\mathrm{RV}$ dysfunction are simply too little, too late and result in only modest transient symptomatic functional improvements with little impact on longer-term survival. There is a concern that with longer follow-up, simple interventions such as ring annuloplasty may not fully protect against residual or recurrent late TR. ${ }^{6}$ Similarly, bioprosthetic tricuspid valve replacement will be associated with a late reoperation due to eventual structural valve deterioration. ${ }^{7}$ With the advances in transcatheter tricuspid technology, the need for reoperative surgery can be mitigated by tricuspid clips, valve-in-ring, and valve-in-valve approaches.

We hope that with their more accurate and complete diagnostic assessments and quantification, studies of new transcatheter tricuspid valve therapeutic technologies will provide clarity, accelerate, and guide ongoing debates by 
heart valve teams on the timing and scope of intervention(s) for individual patients at specific presentations and stages of TR, striving to a long-overdue, more effective clinical balance. ${ }^{6}$

\section{References}

1. Badhwar V, Rankin JS, He M, Jacobs JP, Furnary AP, Fazzalari FL, et al. Performing concomitant tricuspid valve repair at the time of mitral valve operations is not associated with increased operative mortality. Ann Thorac Surg. 2017;103:587-93.

2. Saran N, Dearani J, Said S, Greason K, Pochettino A, Stulak JM, et al. Long-term outcomes of patients undergoing tricuspid valve surgery. Eur J Cardiothorac Surg. 2019;56:950-8.
3. Huttin O, Voilliot D, Mandry D, Venner C, Juillière Y, Selton-Suty C. All you need to know about the tricuspid valve: tricuspid valve imaging and tricuspid regurgitation analysis. Arch Cardiovasc Dis. 2016;109:67-80.

4. Asmarats L, Puri R, Latib A, Navia J, Rodés-Cabau J. Transcatheter tricuspid valve interventions: landscape, challenges, and future directions. J Am Coll Cardiol. 2018;71:2935-56.

5. Fröjd V, Folino G, Jeppsson A, Dellgren G. Mortality after tricuspid valve procedures: a 27-year, single-center experience. J Thorac Cardiovasc Surg. 2021;161: 1239-48.e1.

6. Picichè M, ed. Dawn and Evolution of Cardiac Procedures: Research Avenues in Cardiac Surgery and Interventional Cardiology. Milan: Springer-Verlag Mailand; 2013.

7. McCarthy PM, Bhudia SK, Rajeswaran J, Hoercher KJ, Lytle BW, Cosgrove DM, et al. Tricuspid valve repair: durability and risk factors for failure. J Thorac Cardiovasc Surg. 2004;127:674-85. 